\title{
Amphiphilic Graphene Composite Based on Nanoscale ionic Materials
}

\author{
Liusuo $\mathrm{Wu}^{1,2, a}$, Yueju Zhao ${ }^{1,2, b}$, Jilin Teng ${ }^{1,2}$, Wenhua Miao ${ }^{1,2}$, Huiyu Li ${ }^{1,2}$, \\ Mingjun Sun ${ }^{3}$ \\ ${ }^{1}$ Nari group corporation(state grid electric power search stitute), Beijing, 100070, China \\ ${ }^{2}$ Beijing Guodian Futong science and technology development corporation, Ltd, Bejing, 100070, \\ China \\ ${ }^{3}$ Liaoning Hongyanhe Nuclear power Co., Ltd, Dalian, 100070, China \\ aemail: liusuowu@iccas.ac.cn, bemail:zhaoyj@icass.ac.cn
}

Keywords: Amphiphilic; Graphene; Nanoscale ionic Materials

\begin{abstract}
Sufficient solubility of graphene composite in different solvents is a prerequisite for their further practical applications. In this study, the amphiphilic graphene composite was prepared based on Nanoscale ionic materials (NIMs). The graphene composite showes even dispersion and moths-long stability in deionic water and many oragnic solvents, indicating its amphiphilic nature. The unique properties of the amphiphilic graphene composite hold great potential for practical applications.
\end{abstract}

\section{Introduction}

Among carbonaceous materials, graphene has attracted tremendous research interest due to its unique structural features and outstanding performance[1]. Graphene is a two-dimensional sheet nano-material, which has attracted considerable attention recently because of its unique properties, including superior mechanical strength, as well as high heat and electric conductance[2]. The excellent performances of graphene have great potential application in composite materials. Functional composites based on graphene are famous for higher electric conductivity, so ideally graphene oxide (GO) must be rigorously reduced to $\pi$-conjugation-rich reduced graphene oxide (RGO) to recover the desirable properties. Upon reduction, although graphene can restore the excellent performance, most of the oxygen-containing groups in GO, in particular the hydroxyl and epoxide will be completely removed, so the RGO has so poor solution that hardly well dispersed in many common solvents. Therefore, sufficient solubility and homogeneously dispersion in different solvents is a prerequisite for their further practical applications[3].

Typically, chemical modifications, covalent and non-covalent functionalizations are very common way to improve the dispersibility of graphene[4]. Among these method, chemical modification is a widely used, because the surface of GO have many active group, hydroxyl, epoxy and carboxyl groups. The stable dispersed graphene sheets depend on steric hindrance or electrostatic interactions[5].

Herein, we reported a simple way to prepare amphiphilic graphene composite based on nanoscale ionic materials (NIMs). NIMs, as a kind of organic-inorganic hybrids, are typically composed of an inorganic nanoparticles core covalently grafted with the charged organic corona[6]. Amphiphilic graphene compsosite based on NIMs can stable disperse in many polarity (for example water and ethanol) and non-polarity solvents (such as chloroform and toluene) for about one year[7]. We use the hydroxyl based on graphene oxide as the active group, the process is shown in scheme 1 . First, the sulfonic acid groups were introduced to GO via silane coupling agent of 3-(trihydroxysilyl)-1-propane sulfonic acid (SIT) and hydroxyl. Then, hydrazine reduced silanized graphene oxide (GO-SIT) to RGO-SIT which appears acidity. Finally, reaction of a strong acid in the form of the RGO-SIT with a monoamine functionalized polyetheramine block copolymer, Jeffamine 2070 (M2070) which as a weak base. The final product Amphiphilic graphene compsosites based on NIMs was named RGO-SIT-M2070. In comparison, the excellent amphiphilic was in accordance with its microstructures and inherent property of oligomers. 


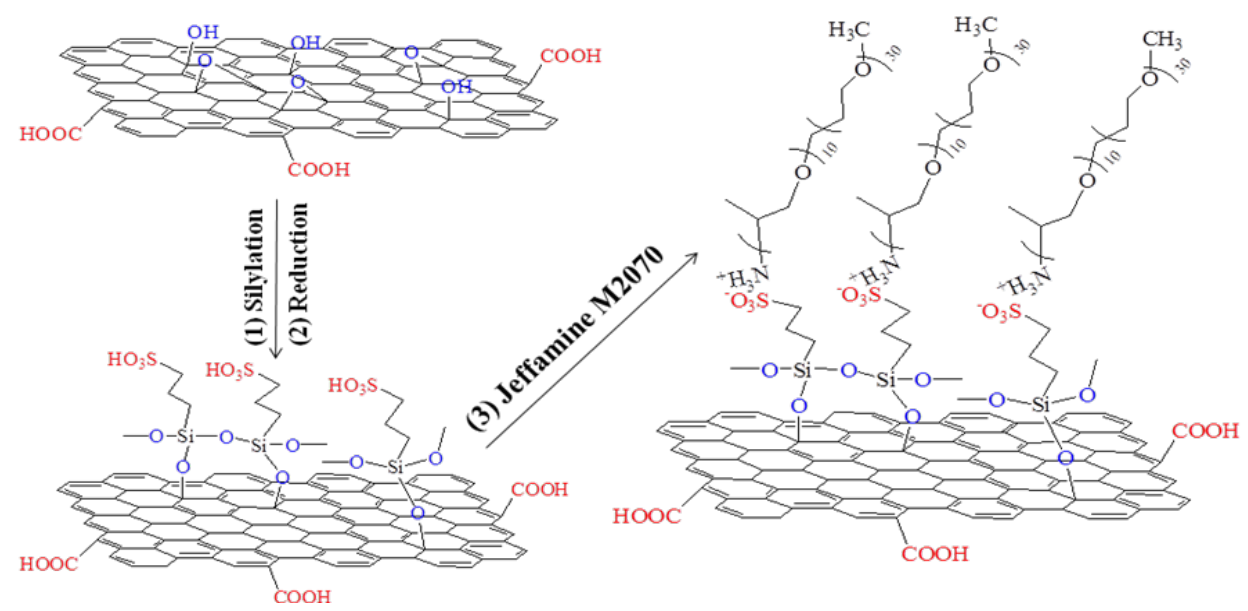

Scheme 1. The synthesis approach and structure of RGO-SIT-M2070

\section{Experiment}

\section{Materials.}

Graphite powder was homemade expandable graphite (220-80 N of Grafguard Inc.), 3-(trihydroxysilyl)-1-propane sulfonic acid (SIT, $40 \mathrm{wt} \%$, Gelest) was purchased from Gelest, Inc. Jeffamine $2070\left(M_{w}=2000\right)$ was kindly supplied by Huntsman. Dialysis membrane (trapped molecular weight $>14000$ ) was bought by JingKeHongDa Biotechnology Co, Ltd. Ion exchange resin was gained from HWRK Chemical company. Other reagents used for reaction and dispersion were all analytically pure.

\section{Preparation of GO.}

In a typical Hummers method[8], expandable graphite powder $(5 \mathrm{~g})$ was put into ice bath $\left(0{ }^{\circ} \mathrm{C}\right)$ with concentrated $\mathrm{H}_{2} \mathrm{SO}_{4}(115 \mathrm{ml})$. KMnO4 was slowly added with stirring about $30 \mathrm{~min}$, keeping this condition 1 hour for further intercalation. Then, the mixture was stirred at $35^{\circ} \mathrm{C}$ for 2 hours. Finally, distilled water $(200 \mathrm{ml})$ was slowly added and maintained statically about 30min, the reaction was terminated by addition of $700 \mathrm{ml}$ distilled water. The mixture was filtered and washed first by $5 \% \mathrm{HCl}$ and then plenty of distilled water. The resulting solid was dried at $50{ }^{\circ} \mathrm{C}$ oven.

\section{Synthesis of RGO-SIT.}

Dispersion of graphene oxide (300 mg GO, $200 \mathrm{ml}$ deionized water) was sonicated for $30 \mathrm{~min}$ followed by the addition of a few drops of $\mathrm{NaOH}$ aqueous solution (10 wt \%) to adjust the $\mathrm{pH}=11$. At the same time 3-(trihydroxysilyl)-1-propane sulfonic acid (SIT, 2g) was diluted with deionized water $(20 \mathrm{ml})$. The GO dispersion was slowly added to the SIT suspension while stirring vigorously. The entire solution was heated to $70{ }^{\circ} \mathrm{C}$ and stirred for $24 \mathrm{~h}$. Then, the suspension was dialyzed using deionized water for $48 \mathrm{~h}$. After dialysis, the functionalized graphene oxide (GO-SIT) was run through an ion exchange column to remove $\mathrm{Na}^{+}$ions and fully protonate the sulfonate groups present. So the protonate GO-SIT dispersion was acidic.

To reduce GO-SIT to RGO-SIT, $0.5 \mathrm{ml}$ 85\% hydrazine monohydrate was added to the dispersion of GO-SIT, then stirred at $80{ }^{\circ} \mathrm{C}$ overnight. Upon reduction, the solution was first filtrated then washed with $5 \% \mathrm{HCl}$ and plenty of deionized water. The product RGO-SIT was dried for further use.

\section{Preparation of Amphiphilic graphene compsosites (RGO-SIT-M2070).}

The dispersion of RGO-SIT was sonicated for $1 \mathrm{~h}$, the final $\mathrm{pH}$ was about 3.5. The RGO-SIT-M2070 was prepared by dissolving a desired amount of Jeffamine 2070 (M2070) in deionized water to a concentration of $10 \mathrm{wt} \%$, then the M2070 solution was added dropwise to the RGO-SIT solution while monitoring the $\mathrm{pH}=10$ of the mixture. The mixture was stirred for $48 \mathrm{~h}$, then the solvent was removed under vacuum at $50{ }^{\circ} \mathrm{C}$ to obtain the tar-like material at the room temperature.

The control sample was prepared by mixing the non-protonated RGO-SIT-Na ${ }^{+}$(Fully protonated 
RGO-SIT react with $\mathrm{NaOH}$ to adjust $\mathrm{pH} \approx 7$ ) directly with M2070 in the same RGO-SIT/M2070 ratio as in the RGO-SIT-M2070.

\section{Characterization.}

The thermal gravimetric analysis (TGA) was performed with a PE Pyris-1 instrument at a heating rate of $10{ }^{\circ} \mathrm{C} / \mathrm{min}$ under $\mathrm{N}_{2}$ atmosphere. The wide angle X-ray diffraction (WAXD) patterns were recorded using a Rigaku D/MAX-RB diffractometer $(40 \mathrm{kV}, 30 \mathrm{~mA})$ with $\mathrm{Cu}-\mathrm{K}_{\alpha}$ radiation with a scanning rate of $4^{\circ} / \mathrm{min}$ for $2 \theta$ from $3^{\circ}$ to $40^{\circ}$. The Atomic force microscopic (AFM) images were recorded with a Bruker Nanoscope V Multimode 8 AFM as the height and phase images were recorded simultaneously. X-ray photoelectron spectroscopy (XPS) measurements were carried out with an ESCALab220i-XL photoelectron spectrometer from VG Scientific. FTIR spectra were recorded using Scientific NICOLET 6700 in the range of $500-4,000 \mathrm{~cm}^{-1}$ with a $4 \mathrm{~cm}^{-1}$ resolution and the coaddition of 30 repetitive scans.

\section{Results and Discussion}

\section{Structure and Component.}
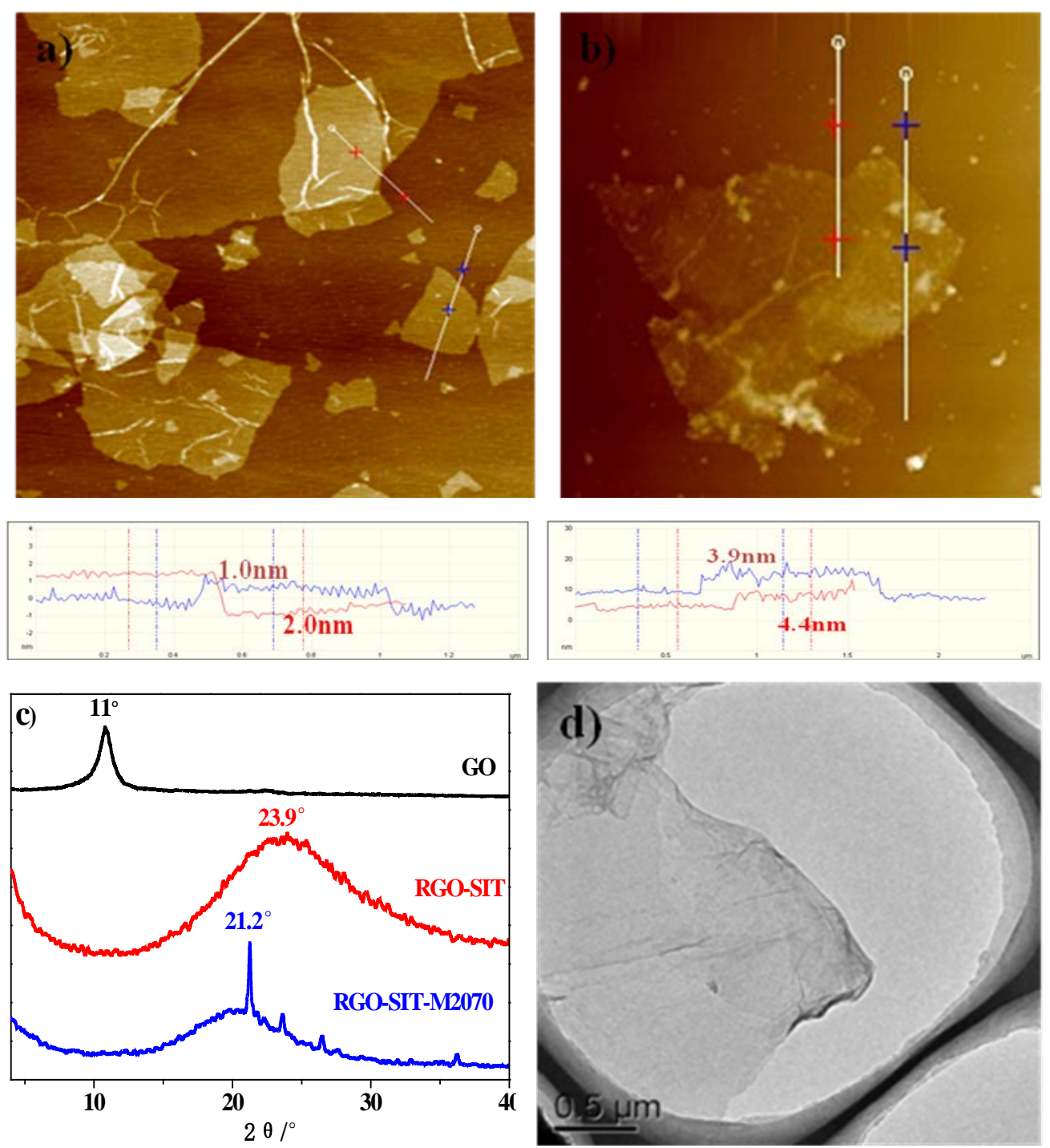

Figure 1 AFM image of a) GO and b) RGO-SIT-M2070. c) XRD patterns of GO, RGO-SIT and RGO-SIT-M2070. d) TEM images of RGO-SIT-M2070

The morphology and structure of GO and RGO-SIT-M2070 samples were studied by tapping-mode AFM, TEM and XRD. Figure 1 a) shows the AFM images of layered GO, the thickness of single-layer is about $1.0 \mathrm{~nm}$ while the double-layer is about $2.0 \mathrm{~nm}$, which is consistent with the result of XRD results (Figure $1 \mathrm{c}$ ) and previous reports. The XRD profile of GO exhibits a 
strong single reflection at $2 \theta=11^{\circ}$, which corresponds to an interlayer $d_{001}$ spacing of $0.8 \mathrm{~nm}$. Upon chemical modification and oligomers grafted, the surface of graphene may have more defects, moreover the thickness increased to about $4 \mathrm{~nm}$ or more (Figure $1 \mathrm{~b}$ ). There are no diffraction peak in the range of $3 \sim 10^{\circ}$, this may be attributed to fully exfoliated so the diffraction peak tends to lower degree $\left(<3^{\circ}\right)$. The RGO-SIT-M2070 can easily disperse in water and exfoliated into thinner layer, a nearly single-layer sheet was observed by the TEM images (Figure $1 \mathrm{~d}$ ).

It is noteworthy that the emergence of peak at $21.2^{\circ}$ of RGO-SIT-M2070 corresponds to pristine graphene, revealing the reduction was occurred in the process of prepare RGO-SIT which has a broad peak at $23.9^{\circ}$. Moreover, the diffraction peak is quiet sharp compared to RGO-SIT, which indicates that the nanosheets have higher order along the stacking direction in the RGO-SIT-M2070.
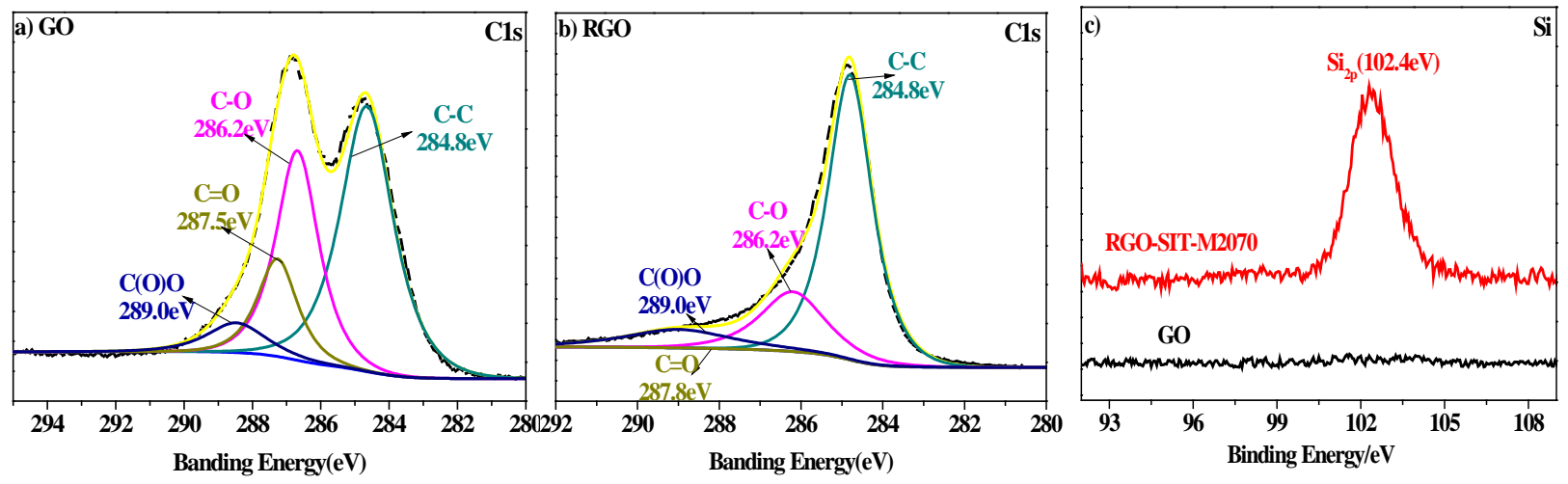

Figure 2 XPS profiles of $\mathrm{C}_{1 \mathrm{~s}}$ for a) GO, b) RGO and $\mathrm{Si}_{2 \mathrm{p}}$ for c) GO and RGO-SIT-M2070

Detailed compositional analysis of GO, RGO and RGO-SIT-M2070 were carried by XPS as shown in Figure 2, which provided the information about the type and surface functionalization of the materials. The C1s-XPS of GO shows four types of carbon bonds, i.e. C-C (284.8 eV) of $s p^{2}$ carbon in the basal plane of GO, others are C-O $(286.2 \mathrm{eV}), \mathrm{C}=\mathrm{O}(287.5 \mathrm{eV})$ and $\mathrm{O}-\mathrm{C}=\mathrm{O}(289.0$ $\mathrm{eV})$. Upon reduction, $\mathrm{C}-\mathrm{C}$ peak becomes dominant, while the oxygen-containing groups become weak (Figure $2 \mathrm{~b}$ ). The $\mathrm{C} / \mathrm{O}$ ratio from 2.2 (GO) to 6.8 (RGO) accompanied by the reduction, also the comparison of XPS spectra of GO and RGO-SIT-M2070 revealed the presence of silicon signal in the RGO-SIT-M2070 sample, which is ascribed to the silane reaction.

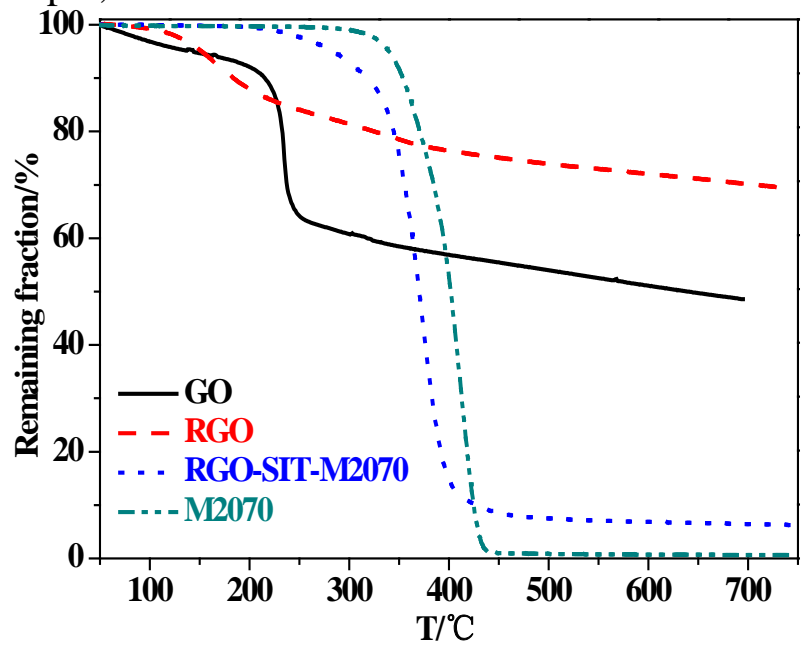

Figure 3 TGA curves of GO, RGO, RGO-SIT-M2070 and M2070

GO, RGO, M2070 and RGO-SIT-M2070 were analyzed by TGA as shown in Figure 3. GO has a major weight loss of $40 \mathrm{wt} \%$ in the temperature range from 50 to $250{ }^{\circ} \mathrm{C}$, which was attributed to the loss of the oxygen-containing functional groups such as $\mathrm{OH}$ and $\mathrm{C}-\mathrm{O}-\mathrm{C}$ groups on the basal planes. The no significant mass loss ranges from 250 to $700{ }^{\circ} \mathrm{C}$ may be ascribed to $\mathrm{C}=\mathrm{O}$ and $\mathrm{COOH}$ with higher thermal stability. The removal of the oxygen-containing groups results in much improved thermal stability of RGO, the weight loss of $15 \mathrm{wt} \%$ compared to $40 \mathrm{wt} \%$ of GO range 
from 50 to $250{ }^{\circ} \mathrm{C}$, the final loss also only $70 \mathrm{wt} \%$ up to $700{ }^{\circ} \mathrm{C}$. The M2070 has no weight loss up to $300{ }^{\circ} \mathrm{C}$, finally leading to its weight loss of $99 \mathrm{wt} \%$ when the temperature reached $440{ }^{\circ} \mathrm{C}$. For RGO-SIT-M2070, no obviously weight loss before $220{ }^{\circ} \mathrm{C}$ compared to $15 \mathrm{wt} \%$ of RGO, which means the weight fraction of RGO in the RGO-SIT-M2070 was quite low, so its decomposition temperature ranges from $220{ }^{\circ} \mathrm{C}$ to $460{ }^{\circ} \mathrm{C}$ with weight loss of $93.8 \mathrm{wt} \%$, which is due to M2070 oligomer. Therefore, the remaining fraction $6.2 \mathrm{wt} \%$ is attributed to the residues of RGO which means the weight fraction of RGO is about $6.2 \mathrm{wt} \% / 0.7=9 \mathrm{wt} \%$ in the RGO-SIT-M2070.

Solubility of amphiphilic graphene composite.

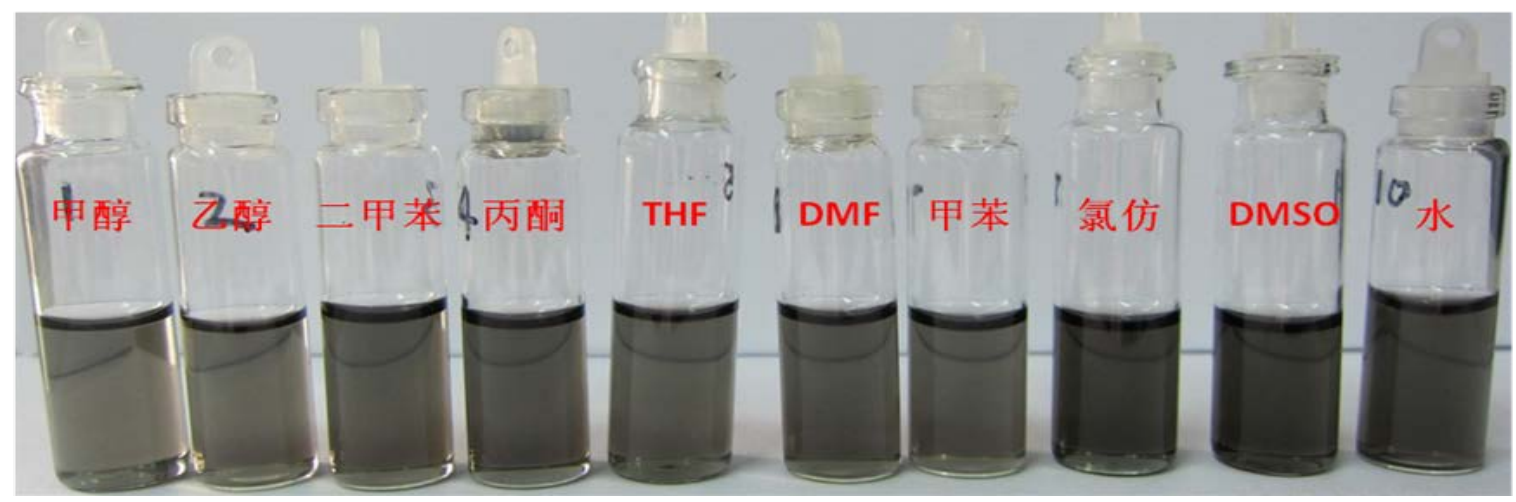

Figure 4 Solubility of the amphiphilic graphene composite in a series of solvents

As we know, there are a few reports on amphiphilic graphene materials. The solubility of graphene materials in desired solvents is demanded in many application areas. As seen from Figure 4 , the prepared amphiphilic graphene composite exhibits good solubility in deionized water and many organic solvents with RGO concentrations of $\sim 0.5 \mathrm{mg} / \mathrm{mL}$, showed exceptional stability over several months. The excellent amphiphilic character of composite was ascribed to the ionic nature of NIMs and the solubility of M2070.

\section{Conclusion}

In summary, we have demonstrated an effective method to prepare amphiphilic graphene based on NIMs. The product exhibits outstanding solubility in many solvents, include deionized water and organic solvents. Both the amphiphilic property of M2070 and the ionic interactions bewteen graphene and M2070 are indispensable for the specific amphiphilic nature of the product. Also, the solubility of graphene composite of can be easily tuned by the stucture of polyetheramine. This will expand the practical application of graphene in many field.

\section{Acknowledgement}

In this paper, the research was sponsored by the Innovative Practical Base of Post doctorate (Young Talents) in Beijing (Project No. 5205E01351DG) and Guodian Futong Corporation Fund of Science and Technology Project (Project No. FTZY 201601).

\section{References}

[1] Singh, V.; Joung, D.; Zhai, L.; Das, S.; Khondaker, S. I.; Seal, S., Graphene based materials: Past, present and future. Progress in Materials Science 2011, 56 (8), 1178-1271.

[2] Kuila, T.; Bose, S.; Mishra, A. K.; Khanra, P.; Kim, N. H.; Lee, J. H., Chemical functionalization of graphene and its applications. Progress in Materials Science 2012, 57, 1061-1105.

[3] Qi, X.; Pu, K. Y.; Li, H.; Zhou, X.; Wu, S.; Fan, Q. L.; Liu, B.; Boey, F.; Huang, W.; Zhang, H., Amphiphilic Graphene Composites. Angewandte Chemie International Edition 2010, 49, 
9426-9429.

[4] Xu, X.; Luo, Q.; Lv, W.; Dong, Y.; Lin, Y.; Yang, Q.; Shen, A.; Pang, D.; Hu, J.; Qin, J., Functionalization of Graphene Sheets by Polyacetylene: Convenient Synthesis and Enhanced Emission. Macromolecular Chemistry and Physics 2011, 212 (8), 768-773.

[5] Zeng, C.; Tang, Z.; Guo, B.; Zhang, L., Supramolecular ionic liquid based on graphene oxide. Physical Chemistry Chemical Physics 2012, 14, 9838-9845.

[6] Fernandes, N. J.; Wallin, T. J.; Vaia, R. A.; Koerner, H.; Giannelis, E. P., Nanoscale Ionic Materials. Chemistry of Materials 2013, 26 (1), 84-96.

[7] Wu, L.; Zhang, B.; Lu, H.; Liu, C.-Y., Nanoscale ionic materials based on hydroxyl-functionalized graphene. Journal of Materials Chemistry A 2014, 2 (5), 1409-1417.

[8] Li, Q.; Dong, L.; Fang, J.; Xiong, C., Property- Structure Relationship of Nanoscale Ionic Materials Based on Multiwalled Carbon Nanotubes. ACS Nano 2010, 4 (10), 5797-5806. 